\title{
Dynamic Programming QoS-based Classification for Links with Limited Service Levels
}

\author{
Amr Mohamed ${ }^{1}$ and Hussein Alnuweiri ${ }^{1}$ \\ ${ }^{1}$ Department of Electrical \& Computer Engineering, 2356 Main Mall, \\ University of British Columbia, Vancouver, B.C. Canada, V6T 1Z4 \\ Emails: \{amrm, hussein\}@ece.ubc.ca
}

\begin{abstract}
We investigate the QoS-based classification of traffic streams for a multi-class link model with predetermined service levels. Specifically, we consider a link model with fixed service levels or fixed class weights which may be represented by a finite number of MPLS Label-Switched-Paths (LSPs). Our target is to classify a set of traffic streams each with arbitrary local QoSdemand into a small number of service levels while optimizing the residualallocated-resources as a result of the traffic classification. The residualallocated-resources will be measured by the service-quantization-overhead which is the summation of the differences between the required QoS and the offered service level for all traffic streams. We formulate the classification as a Dynamic-Programming problem. We then present a group of polynomial-timealgorithms to obtain the optimal classification for soft and hard QoS requirements. We also present the concept of "differentiation factor" and show the effect of this factor on minimizing the quantization-overhead.
\end{abstract}

\section{Introduction}

The proliferation of differentiated services has been one of the rising challenges to service providers in order to support cost-effective, large scale networks with diverse applications and services. On the other hands, differentiated services promote the idea of supporting limited service levels to provide QoS while maintaining the network scalability. This idea has wide range of acceptance by some applications. However, some emerging applications and services such as MPLS tunnels, Virtual Private Networks (VPNs), Overlay networks, and multicast-based QoS networks, may inherently restrict the aggregation of traffic streams in network nodes. Nonetheless, they still require different levels of guarantees at each network node in order to guarantee the end-to-end QoS. Therefore, the support of per-flow QoS in a network environment with finite set of service levels and traffic classes has been the subject of extensive research[1][2].

To provide the required end-to-end QoS, many resource reservation mechanisms have been discussed in the literature and can be used to partition the end-to-end QoS into local QoS requirements on each network element (link)[4]. However, for a network framework that supports a limited set of service levels at each network link, 


\section{Dynamic Programming QoS-Based Classification}

the local QoS required for each connection or flow will be quantized based on these service levels. Assigning service level which provides higher QoS than the required will lead to waste of network resources while, assigning service level with lower QoS than the required may not be accepted by the application or the service agreement with the network user. Therefore, an important network optimization problem is how to assign the network connections to the best service level that minimizes the total quantization overhead. Such an overhead is the quantization penalty that might have to be incurred by either the user or the service provider. Hence, addressing this problem will have a large significance especially for high speed links (e.g. optical links) that may potentially carry large number of connections.

The problem of obtaining optimal quantized service levels for a set of connection requests has been investigated before in [3], [5], and [6]. Rouskas et al. in [3] defined a similar framework of assigning MPLS tunnels with specified data rates to a set of quantized service levels such that the performance penalty of wasted bandwidth is minimal. The authors in [5], and [6] discussed the problem in a different context where the set of requests are group of receivers on a multicast tree. The present study offers solutions to the generalized problem where connections have both rate and QoS requirements and presents polynomial time algorithms for fixed service levels or predetermined class weights link model for soft and hard QoS requirements.

\section{Model and Problem Formulation}

\subsection{Model}

We assume a network element (link) that offers a set of service levels $S_{L}=\left\{x_{1}, x_{2}, \ldots, x_{L}\right\}$ and has a link capacity $C$. Typically, the service levels are dependent on the link capacity according to the inherent model of the link and the characteristics of the incoming traffic streams. The $l$-th level corresponds to a connection with QoS of $x_{l}$ given that the bandwidth requirement for this connection is $\leq C$. We also have a set of $N$ connections $\mathfrak{R}=\left\{r_{1}, r_{2}, \ldots, r_{N}\right\}$. Each connection request is defined using both bandwidth demand $d_{i}$ and one local QoS requirement $Q_{i}$ (e.g. average delay, dropping probability, jitter, etc.), $r_{i} \equiv\left\{d_{i}, Q_{i}\right\}$. We assume that the connection requests are sorted based on the local QoS requirements in non-decreasing order, (i.e. $Q_{1} \leq Q_{2} \leq Q_{3} \ldots \ldots \leq Q_{N}$ ).

We also define a utility function $U\left(Q_{i}, x_{l}\right)$ which measures the quantization penalty which is proportional to the difference between the achieved and offered QoS. Notice that if $x_{l}$ is better than $Q_{i}$, this will result in a waste of allocated resources whereas if $x_{l}$ is worse than $Q_{i}$, this may have a disadvantage for the application generating the 
traffic stream. Therefore, we will consider the general case where $U\left(Q_{i}, x_{l}\right)$ measures the penalty in both scenarios. The following are some common examples.

The absolute difference $U\left(Q_{i}, x_{l}\right)=\left|Q_{i}-x_{l}\right|$,

The logarithmic difference function $U\left(Q_{i}, x_{l}\right)= \begin{cases}\log \left(\left|Q_{i}-x_{l}\right|\right) & Q_{i} \neq x_{l} \\ 0 & \text { Otherwise }\end{cases}$

\subsection{Proportional Differentiation Model}

As part of the classification model, we consider a link which satisfies the proportional differentiation model (PDM) for traffic scheduling with fixed set of weights and finite number of traffic classes. Such a model has been adopted by several proposed mechanisms in different types of networks like [7], and [8]. The PDM dictates the following relationship for all pairs of service classes.

$$
\frac{x_{i}(t, t+\tau)}{x_{j}(t, t+\tau)}=\frac{\omega_{i}}{\omega_{j}}
$$

Where $x_{i}$, and $x_{j}$ are the service levels (e.g. average delay, dropping probability, etc.) achieved for two classes $i$ and $j$ in the time period $\tau$, and $\omega_{i}$ and $\omega_{j}$ are the weights assigned to these classes. We consider connection requests with life time long enough for equation (3) to be valid. See [10] for details about how to calculate the service levels.

\subsection{Optimization Problem}

The optimization problem, generally, is to classify $N$ connection requests $\Re=\left\{r_{1}, r_{2}, \ldots, r_{N}\right\}$ to one of the $L$ service levels $S_{L}=\left\{x_{1}, x_{2}, \ldots, x_{L}\right\}$ while minimizing the quantization overhead. The classification process is shown in Figure 1. Formally, we need to find the set of $L$ classified groups $\pi_{L}^{*}=\left\{G_{1}^{*}, G_{2}^{*}, \ldots, G_{L}^{*}\right\}$ such that $\psi\left(\pi_{L}^{*}\right) \leq \psi\left(\pi_{L}\right)$ $\forall \pi_{L}$ where $\psi\left(\pi_{L}\right)$ measures the quantization overhead and is defined as follows:

$$
\psi\left(\pi_{L}\right)=\sum_{l=1}^{L} \sum_{r_{i} \in G_{l}} U\left(Q_{i}, x_{l}\right)
$$

Each connection request has to be assigned to exactly one service level. The meaning of the objective function dictated by equation (4) is that by minimizing the quantization overhead as a result of the classification process, we guarantee that the connection request is assigned a QoS value which is as close as possible to the required. Hence, the service provider is guaranteed the best resource utilization with minimum or no violation to the QoS required by the application.

We consider a subset of the possible classification policies where the connection requests are sorted based on the QoS requirements in a non-decreasing order, (i.e. $\left.Q_{1} \leq Q_{2} \leq Q_{3} \ldots . . \leq Q_{N}\right)$. This is called ordered classification. 
Ordered Classification - A group set $\pi_{L}=\left(G_{1}, . ., G_{L}\right)$ is an ordered classification if $\forall r_{i} \in G_{a}$ and $\forall r_{j} \in G_{b}, Q_{i} \leq Q_{j}$, when $x_{a}<x_{b}$.

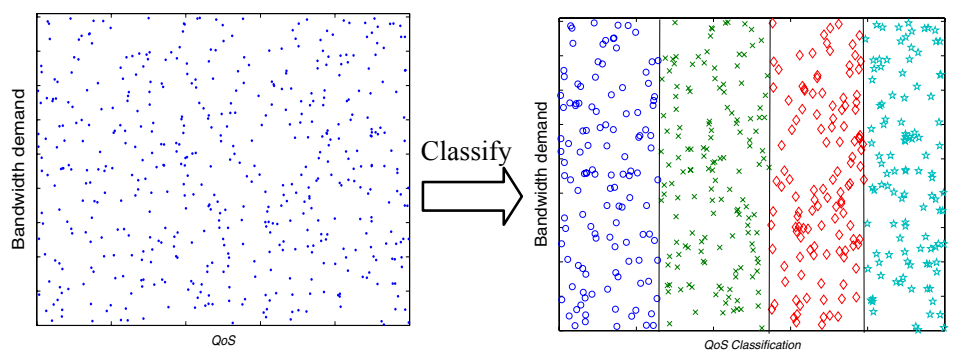

Figure 1. QoS-based classification

This is also shown in Figure 1 that the groups are based on the order of the values of $Q_{i} \quad i=1, \ldots, N$. The assumption of considering only the ordered classifications is valid if and only if the utility function fulfills the following property (see theorem 1 in [5]).

Utility Property - Intuitively, the utility function is non-increasing in the interval $Q_{i} \in\left[0, x_{l}\right]$ and it is non-decreasing in the interval $Q_{i} \in\left[x_{l}, \infty[\right.$.

Indeed, the assumption of considering only the ordered classifications will limit the number of possible solutions for optimality. However, in [10], we proved that Brute Force technique using ordered classification policy will lead to an exponential running time solution.

\section{Optimal solution for fixed set of service levels and soft QoS requirements}

For a set of service levels $S_{L}=\left\{x_{1}, x_{2}, \ldots, x_{L}\right\}$ which may be based on the link's total load, and a set of QoS requests $\varphi_{N}=\left\{Q_{1}, Q_{2}, \ldots, Q_{N}\right\}$, we form the system matrix $A_{N \times L}$ such that the elements of $A, a_{i j}=U\left(Q_{i}, x_{j}\right) i=1, \ldots, N$, and $j=1, \ldots, L$.

To minimize $\psi\left(\pi_{L}\right)$ in a tractable model, we map the problem to the following dynamic program.

$$
\psi(i, l)=\psi(k, l-1)+\sum_{j=k+1}^{i} a_{j l}
$$

Where $\psi(i, l)$ is the local optimal quantization overhead for requests up to request $i$ using $l$ service levels. This local optimal value is calculated based on recursive enumeration using dynamic program FSL_OPT_CLASSIFY shown in Figure 2.

Complexity of calculating $a_{i j}$ for $i=1, \ldots, N$ and $j=1, \ldots, L$ is $O(N L)$. The summation of $a_{i j}$ for $i=1, \ldots, N$ and $j=1, \ldots, N$ may be pre-computed with a complexity of $O\left(N^{2} L\right)$ (similar pre-computation is explained in details in [5]). The complexity of algorithm 
Dynamic_Program's main loop (line 1 to 7) is also $O\left(N^{2} L\right)$. Therefore, the complexity of FSL_OPT_CLASSIFY is $O\left(N^{2} L\right)$.

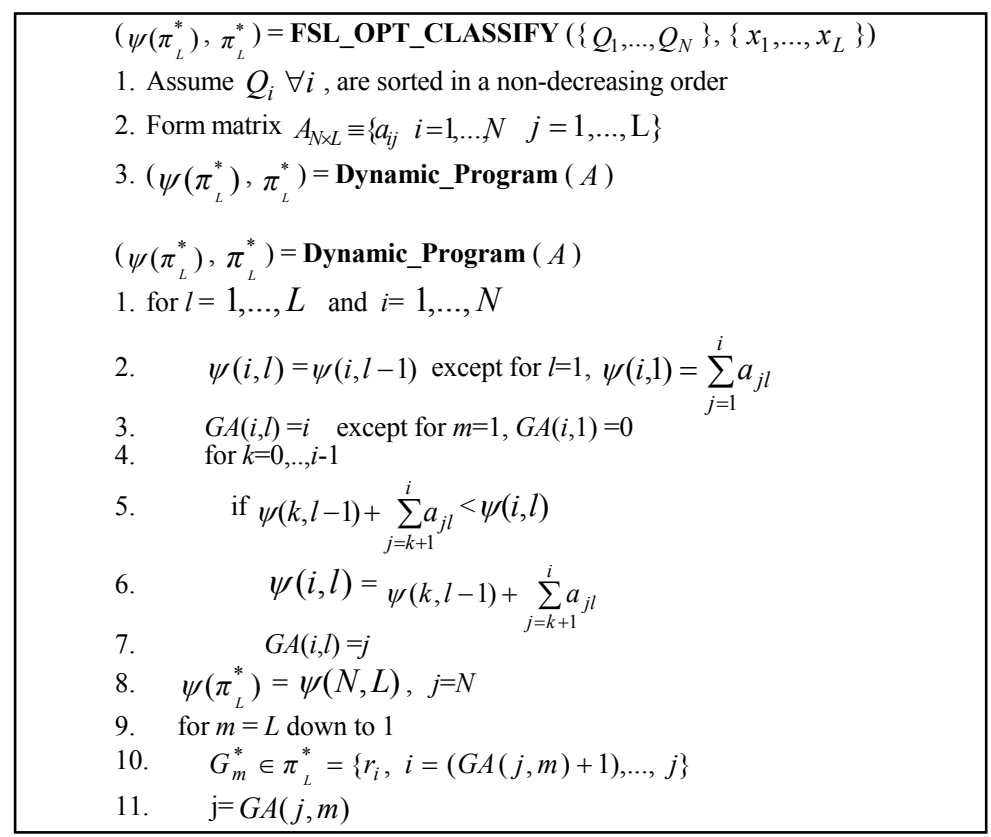

Figure 2. Optimal classification with fixed service levels

\section{Optimal Solution with Admission Control (Hard QoS)}

Up till now, we assume that the incoming traffic streams can tolerate service levels with lower value of QoS than the QoS required by each stream. Although, this might be tolerable by some applications, an admission control mechanism might impose some restrictions on the minimum level of service achieved by the traffic streams. If the link cannot provide the traffic streams with a level of service higher than a certain limit, the admission control mechanism might reject the addition of new traffic streams. In order to capture this fact, we introduce a new variable called tolerance factor $\delta$. This factor represents the maximum percentage of QoS drop tolerated by the traffic stream. If $\delta=0$, this means that the traffic stream cannot tolerate any service drop. Also, each traffic stream might impose different tolerance factor based on the type of application generating its traffic. Therefore, we will assume the general case that each traffic stream has a different tolerance factor (i.e. $r_{i} \equiv\left\{d_{i}, Q_{i}, \delta_{i}\right\}$ ). We then want to guarantee that:

$$
\left(\left(1+\delta_{i}\right) Q_{i}-x_{j}\right) \geq 0 \quad i=1, \ldots, N \quad j=1, \ldots, L
$$


Which means that the achieved QoS $x_{j}$ must not be worse than the required QoS $Q_{i}$ by more than a percentage of $\delta_{i}$ of the required QoS $Q_{i}$.

To incorporate this condition in the FSL_OPT_CLASSIFY algorithm we will exclude the assignments where condition (5) is not satisfied by setting $a_{i j}=\infty$ as shown in Figure 3.

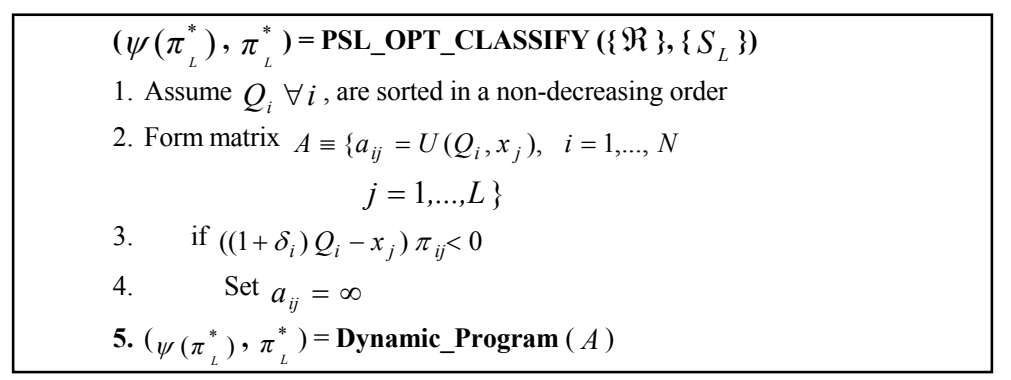

Figure 3. Optimal classification for arbitrary $\delta$

\section{Class Weights Service Differentiation}

Based on PDM model explained in section 2.2, we will first assume that the link capacity condition is not enforced or there is a light traffic load on the link. Obviously, if the link is lightly loaded, the QoS achieved by the traffic streams may potentially exceed the QoS requirements. In this case, we have some flexibility to assign the traffic streams to the different service classes. To do that, we will follow the same methodology explained in section 3. This time, we will use $a_{i j}=U\left(Q_{i}, \gamma \omega_{j}\right)$ such that $\omega_{j}$ is the weight assigned to service class $j, j=1, . ., L$, and $\gamma$ is the service differentiation factor selected based on the distribution of the incoming QoS requests.

Ideally, we need to select the value of $\gamma$ such that $\psi\left(\pi_{L}^{*}\right)$ has the lowest value possible. However, this means that we need to try all possible values of $\gamma$ and get the optimal classification for each case, and select $\gamma$ such that the value of $\psi\left(\pi_{L}^{*}\right)$ is minimized which has a very high complexity. Instead, in the following, we introduce two heuristic estimates for $\gamma$. Then, we will drive the optimal value of $\gamma$ that guarantees a minimum value for $\psi\left(\pi_{L}^{*}\right)$. First, we will present the following lemma.

Lemma 1 - If $U\left(Q_{i}, \gamma \omega_{j}\right)$ is non-increasing in the interval $\left[0, \gamma \omega_{j}\right]$ and nondecreasing in the interval $\left[\gamma \omega_{j}, \infty\left[\right.\right.$, and $U\left(Q_{i}, \gamma \omega_{j}\right)=C_{i j} * U\left(Q_{i} / \omega_{j}, \gamma\right)$, where $C_{i j}$ is any constant, then, in order to minimize the value of $\psi\left(\pi_{L}^{*}\right), \gamma$ must be selected in the range $\left[\min \left(Q_{i} / \omega_{j}\right), \max \left(Q_{i} / \omega_{j}\right)\right] \quad i=1, \ldots, N \quad j=1, \ldots, L$. 
See the proof in [11]. This lemma limits the scope of possible values for $\gamma$ between the minimum and maximum possible weighted QoS values.

First Heuristic estimation for $\gamma$ - One natural selection for the value of $\gamma$ is the center of the sample data $d_{i j}=Q_{i} / \omega_{j}$, or the statistical median $\left(d_{i j}\right) i=1, \ldots, N$ and $j=1, \ldots, L$. The intuition behind this selection is to try to lower the values of $U\left(Q_{i}, \gamma \omega_{j}\right)$ by selecting $\gamma$ in the center of $d_{i j}$ to minimize the value of $\psi\left(\pi_{L}^{*}\right)$. The complexity of calculating the median is $O(N L)$ [9].

Second Heuristic estimation for $\gamma$ - Another heuristic estimation for the value of $\gamma$ is to select it such that the total $\sum_{i=1}^{N} \sum_{j=1}^{L} a_{i j}$ is minimized. The intuition here is if we minimize this total, then we may potentially lower the value of the sum on any subset of $i, j$. Although, we have infinite values of $\gamma$ in this case to try, the following theorem limits the possible values of $\gamma$ given some conditions on the utility function.

Theorem 1 - If $U\left(Q_{i}, \gamma \omega_{j}\right)$ is a piecewise concave function, and $U\left(Q_{i}, \gamma \omega_{j}\right)=C_{i j} * U\left(Q_{i} / \omega_{j}, \gamma\right)$ where $C_{i j}$ is constant, then $\gamma$ must take one of the values of $d_{i j}=Q_{i} / \omega_{j} \quad i=1, \ldots, N$ and $j=1, \ldots, L$ in order to minimize the summation $\sum_{i \in I} \sum_{j \in J} a_{i j}$ for any subset of $i$, and $j$.

This theorem implies that to get the value of $\gamma$ that minimizes the summation $\Psi=\sum_{i=1}^{N} \sum_{j=1}^{L} a_{i j}$, we only need to check $N^{*} L$ possible values of $\gamma \in\left\{d_{i j}=Q_{i} / \omega_{j}, \quad i=1, \ldots, N j=1, \ldots, L\right\}$, given that $U\left(Q_{i}, \gamma \omega_{j}\right)$ is a piecewise concave function and the condition $U\left(Q_{i}, \gamma \omega_{j}\right)=C_{i j} * U\left(Q_{i} / \omega_{j}, \gamma\right)$ is satisfied. See the proof in [11]. The piecewise concave function means that $U\left(Q_{i}, \gamma \omega_{j}\right)$ is non-increasing concave in the interval $\left[0, \gamma \omega_{j}\right]$ and non-decreasing concave in the interval $\left[\gamma \omega_{j}, \infty[\right.$. Some examples for piecewise concave functions are defined by equations (1) and (2).

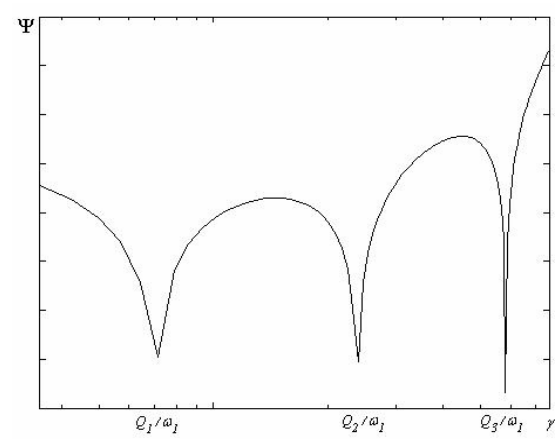

Figure 4. The effect of $\gamma$ for $3 Q_{i}{ }^{\prime} s$ and one $\omega_{j}$ 
To illustrate the effect of the last theorem, Figure 4 shows the summation $\Psi$ for all possible values of $\gamma$ using $U\left(Q_{i}, \gamma \omega_{j}\right)$ as defined by equation (2) for three incoming traffic streams and one class of service. It is clear from the figure, that the possible minimum values of $\Psi$ correspond to the values of $\gamma \in\left\{Q_{1} / \omega_{1}, Q_{2} / \omega_{1}, Q_{3} / \omega_{1}\right\}$.

Given the result of Theorem 2, we get the value of $\gamma$ that minimizes $\Psi$ as shown by the algorithm min_sum_df in Figure 5. Complexity of this algorithm is $O\left(N^{2} L^{2}\right)$ assuming the summation on line 3 is $O(N L)$.

Theorem 1 also implies that to get the value of $\gamma$ that minimizes $\psi\left(\pi_{L}^{*}\right)$ we can substitute $\gamma$ with one of the values $d_{i j}$ and get the optimal classification for each possible value of $\gamma$, and finally select the value of $\gamma$ that has the minimum $\psi\left(\pi_{L}^{*}\right)$. This is illustrated by algorithm min_opt_df in Figure 6. Notice that the optimal classification $\pi_{L}^{*}$ may change by changing the value of $\gamma$. However, Theorem 2 states that algorithm min_opt_df finds the optimal $\gamma$. The proof is in [11].

Theorem 2 - Algorithm min_opt_df finds the optimal $\gamma^{*} \in$ $\left\{d_{i j}=Q_{i} / \omega_{j}, i=1, \ldots, N j=1, \ldots, L\right\}$ such that $\psi\left(\pi_{L}^{*}\left(\gamma^{*}\right), \gamma^{*}\right) \leq \psi\left(\pi_{L}^{*}(\gamma), \gamma\right) \forall \gamma$ if $U\left(Q_{i}, \gamma \omega_{j}\right)$ is a piecewise concave function, and $U\left(Q_{i}, \gamma \omega_{j}\right)=C_{i j} * U\left(Q_{i} / \omega_{j}, \gamma\right)$ where $C_{i j}$ is any constant.

By replacing line 6 of algorithm min_sum_df with a call to the PSL_OPT_CLASSIFY algorithm, we get the value of $\gamma$ that minimizes $\psi\left(\pi_{L}^{*}\right)$ as shown in Figure 6 .

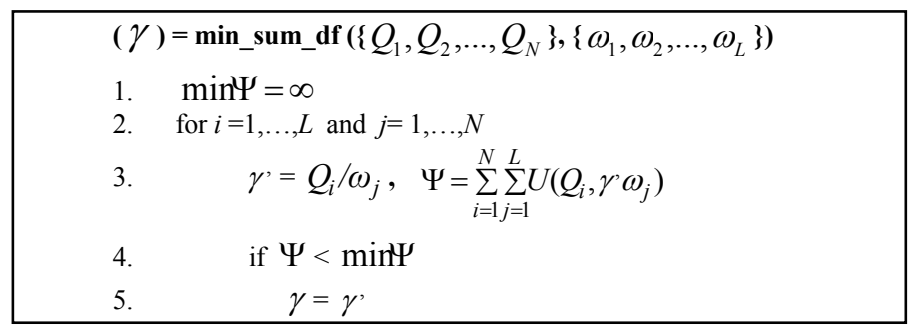

Figure 5. Minimum $\Psi$ Differentiation factor

\begin{tabular}{|c|}
\hline $\begin{array}{l}(\gamma)=\text { min_opt_df }\left(\{\mathfrak{R}\},\left\{\omega_{1}, \omega_{2}, \ldots, \omega_{L}\right\}\right) \\
\text { 1. } \quad \min \Psi=\infty \\
\text { 2. } \quad \text { for } i=1, \ldots, L \text { and } j=1, \ldots, N \\
\text { 3. } \quad \gamma=Q_{i} / \omega_{j}, \quad S_{L}=\left\{\gamma^{\prime} \omega_{\mathrm{j}}, j=1, \ldots, L\right\} \\
\text { 4. } \quad \Psi=\text { PSL_OPT_CLASSIFY }\left(\{\Re\},\left\{S_{L}\right\}\right) \\
\text { 5. } \quad \text { if } \Psi<\text { min } \Psi \\
6 . \quad \gamma=\gamma,\end{array}$ \\
\hline
\end{tabular}

Figure 6. Minimum $\psi\left(\pi_{L}^{*}\right)$ differentiation factor 
From section 4, the complexity of PSL_OPT_CLASSIFY is $O\left(N^{2} L\right)$. So, the complexity of this algorithm is $O\left(N^{3} L^{2}\right)$.

\section{Class weights Service differentiation with limited link capacity}

The link capacity $C$ will impose restrictions on the values of service differentiation used. Our assumption her is that relationship between the capacity and the service differentiation factor is known through statistical modeling (e.g. like the model described in section 2.2) or through numerical estimation. From this relationship, we can obtain the minimum value of $\gamma$, or $\gamma_{\min }$ (line 2 in Figure 7), which can be used to modify the algorithm min_opt_df to obtain the optimal classification for $\forall \gamma \geq \gamma_{\min }$. The modified algorithm is shown in Figure 7. The complexity here is also $O\left(N^{3} L^{2}\right)$.

The algorithm uses the result of theorem 2 and selects a subset of possible values of $\gamma^{*} \in\left\{d_{i j}=Q_{i} / \omega_{j}, \quad i=1, \ldots, N \quad j=1, \ldots, L\right\}$ which are greater than $\gamma_{\min }$ where $\gamma_{\min }$ is imposed by the limited link capacity.

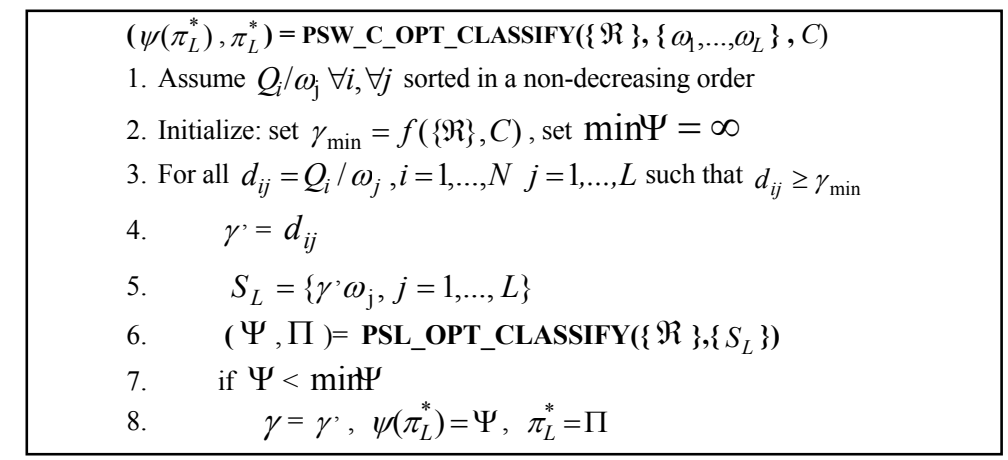

Figure 7. Predetermined class weights classification with limited link capacity

\section{Experimental Evaluation}

In this section we examine the normalized quantization overhead defined as:

$$
\psi_{n}\left(\pi_{L}^{*}\right)=\psi\left(\pi_{L}^{*}\right) / \sum_{i=1}^{N} Q_{i}
$$

We measure this overhead in different scenarios using the algorithms explained before. We take the link's dropping probability as an example for QoS. We assume, as example, the model explained in section 2.2 when it is relevant. 
We present first the results for variable sized sets of connection requests. For each set, the dropping probability is distributed from $10^{-1}$ to $10^{-10}$. The class weights are selected sequentially from the set $W=\left\{10^{-1}, 10^{-2}, \ldots, 10^{-10}\right\}$ such that for $L=2$, we use only the first 2 weights, for $L=3$, we use the first 3 and so on. Figure 8 and Figure 9 show the results using algorithm FSL_OPT_CLASSIFY for uniform distribution values of dropping probability when the link is fully loaded (i.e. link load $\approx 1$ ). Each point on the figures is taken from the average of 10 sets of dropping probability values each with size $N$ as shown. For Figure 8, we notice that the increase in the normalized overhead is insignificant for different values of $N$ and even by increasing the number of service levels. This in fact should be an indication that the class weights are not selected efficiently. This is depicted by Figure 9 where the class weights are selected from the set $W=\left\{2^{-1}, 2^{-2}, \ldots, 2^{-8}\right\}$. We notice, in this case, that the change in overhead by increasing the service levels could reach $30 \%$ when the weights are selected properly and the minimum quantization overhead can become as low as $16 \%$ using 5 service levels. However, even in this case, it is clear that the gain of decreasing the normalized overhead is not significant after $L=5$. In [10], we showed that for clustered QoS distributions, the quantization overhead can be drastically reduced using proper class weights.

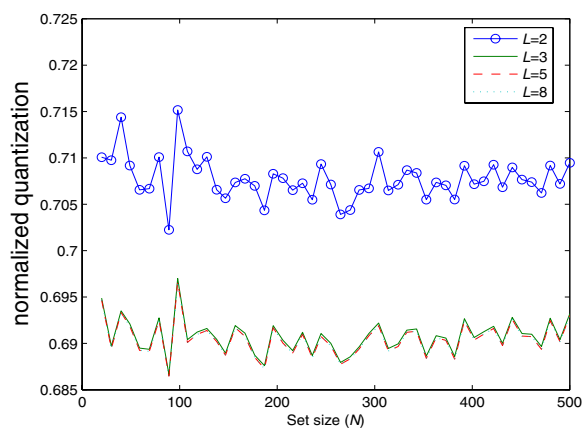

Figure 8. Variable sized sets for $W=\left\{10^{-1}, 10^{-2}, \ldots, 10^{-8}\right\}$

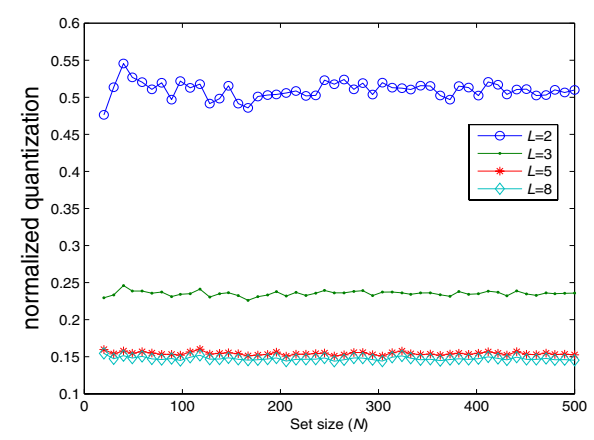

Figure 9. Variable sized sets for $W=\left\{2^{-1}, 2^{-2}, \ldots, 2^{-8}\right\}$

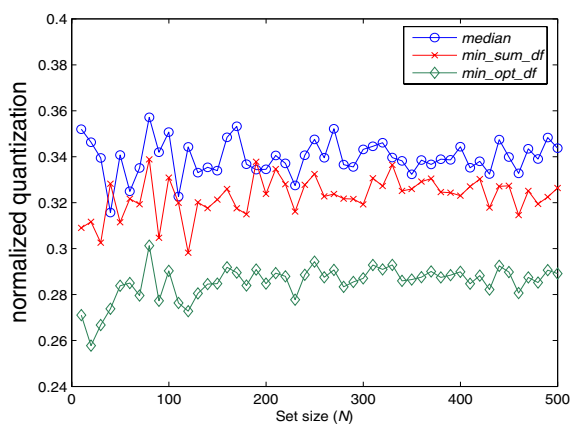

Figure 10. $\gamma$ estimation methods with uniform distribution

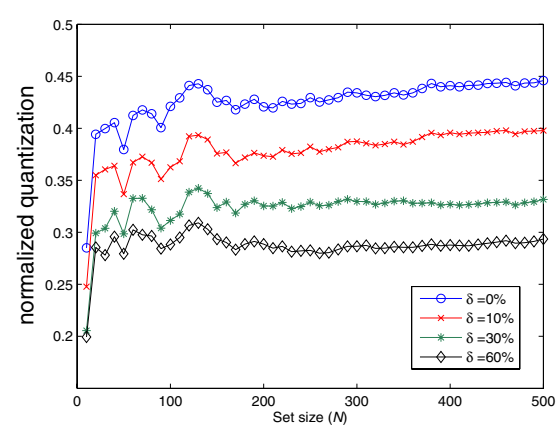

Figure 11. Effect of tolerance factor for different set size

(C)GESTS-Oct.2005 
Figure 10 and Figure 11 show the results for variable service levels based on fixed class weights and variable $\gamma$ as explained in section 5. Figure 10 shows the effect of different estimation methods of $\gamma$ on the normalized quantization overhead for uniformly distributed QoS values. We see that the estimation of $\gamma$ using the min_sum_df algorithm performs slightly better than the median in case of uniform distribution. The estimation using the min_opt_df performs consistently better than the 2 other methods. Figure 11 shows the tolerance effect for uniformly distributed QoS values using the PSW_C_OPT_CLASSIFY algorithm with 4 service levels when the link is almost fully loaded. Clearly, increasing the tolerance factor plays a significant role in decreasing the quantization overhead regardless of the number of traffic streams. However, increasing the tolerance factor is limited by the type of application generating the traffic streams.

\section{Conclusion and Future Work}

We have presented a group of algorithms for calculating the optimal classification for a set of traffic streams with diverse QoS requirements for a link model with a predetermined service levels or predetermined class weights. Our results show the effect of selecting the class weights based on the statistical distribution of the incoming connection requests. It was shown that by carefully selecting the class weights, the quantization overhead can be significantly reduced especially for number of service levels in the range $[3,5]$. The results also show that the effect of increasing $L$ diminishes as it rises more than 5 . We also proposed three different methods for calculating the differentiation factor and discussed the effect of each method on the quantization overhead.

We are currently extending our solutions to include the case of a link model with self adjustable service levels or class weights. In this case, we are supposed to allocate the service levels freely such that the partitioned groups minimize the quantization overhead.

\section{References}

[1] P. Chaporkar, J. Kuri, "A Network Architecture for Providing Per Flow Delay Guarantees with Scalable Core", Journal for High Speed Networks, vol. 12, no. 3,4, pp. 87-109, Jan, 2002.

[2] Mei Yang, Yan Huang, Jaime Kim, Meejeong Lee, Tatsuya Suda and Mastubara Daisuke, "An End-toEnd QoS Framework With On-Demand Bandwidth Reconfiguration", IEEE INFOCOM, Mar, 2004.

[3] George N. Rouskas, Laura E. Jackson, "Optimal Granularity of MPLS Tunnels", In Proceedings of the 18th International Teletraffic Congress (ITC-18), pp. 1-10, August 31-September 5, 2003, Berlin, Germany.

[4] D. H. Lorenz, A. Orda, "Optimal Partition of QoS Requirements on Unicast Paths and Multicast Trees", IEEE/ACM Transactions on Networking, Vol. 10, No. 1, pages 102-114, February 2002.

[5] Y. Richard Yang, Min Sik Kim, Simon S. Lam, "Optimal Partitioning of Multicast Receivers", Proceedings of the 8th IEEE ICNP, 2000.

[6] T. Jiang, M. H. Ammar, and E. W. Zegura. "On the use of destination set grouping to improve interreceiver fairness for multicast ABR sessions", In Proceedings of IEEE INFOCOM, 2000. 
[7] Y. Chen, M. Hamdi, and D. H. K. Tsang, "Proportional QoS over OBS networks", in Proceedings of IEEE Globecom 2001, NY, pp.1510-1514.

[8] N. Christin, J. Liebeherr and T. Abdelzaher, "A Quantitative Assured Forwarding Service”, In Proceedings of IEEE INFOCOM 2002, vol. 2, pages 864-873. New York, NY. June 2002.

[9] S. Battiato, D. Cantone, D. Catalano, G. Cincotti, M. Hofri, "An efficient algorithm for the approximate median selection problem", Proceedings of the Fourth Italian Conference, CIAC 2000, Rome, Vol. 1767, 2000, pp.

[10] A. Mohamed, H. Alnuweiri, "Optimal QoS-based classification for link models with predetermined service levels", Proceedings of the 8th International Conference on Telecommunications, Contel 2005.

[11] A. Mohamed, H. Alnuweiri, "Dynamic Programming QoS-based Classification for Links with Limited Service Levels", To be published in LCN 2005.

\section{Biography}

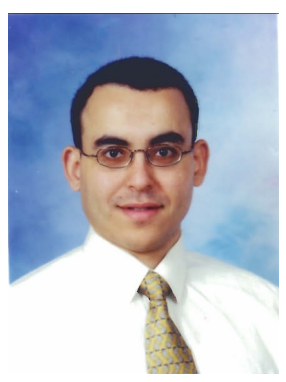

A Name: Amr Mohamed

Address: Department of Electrical \& Computer Engineering (ECE), 2356 Main Mall, University of British Columbia (UBC), Vancouver, B.C. Canada, V6T $1 Z 4$

Education \& Work experience: Amr Mohamed received his M.S. in electrical and computer engineering from UBC, Vancouver, Canada, 2001. He received his B.S. from Cairo University in 1993. Since $1994 \mathrm{Amr}$ has been working as a Research and Teaching Assistant at the National Telecommunication Institute, Cairo, Egypt. His main research interests include, QoS and resource allocation for WDM and wireless Networks, videoconferencing, video Broadcast/Multicast.

Tel: +1-604-222-0484 E-mail: amrm@ece.ubc.ca

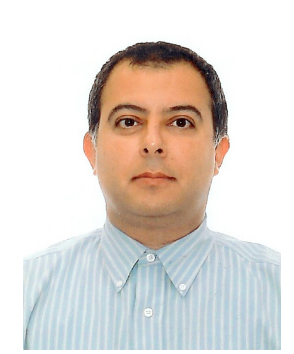

$\Delta$ Name: Hussein Alnuweiri

Address: Department of Electrical \& Computer Engineering (ECE), 2356 Main Mall, University of British Columbia (UBC), Vancouver, B.C. Canada, V6T 1 Z4

Education \& Work experience: Hussein M. Alnuweiri obtained his Ph.D. Degree in 1989 from the University of Southern California, Los Angeles. He is currently a Professor in the ECE Department at UBC. His research interests cover all aspects of traffic engineering and quality-of-service mechanisms in wired and wireless packet networks including constraint-based routing, scheduling algorithms, and real-time multimedia communications. He has represented UBC at the ATM-Forum, and is a delegate of the Standards Council of Canadian for the MPEG-4 (ISO/IEC JTC 1/SC 29/WG 11) committee. Dr. Alnuweiri has authored or co-authored over 100 journal and conference papers, and holds three US and International patents.

Tel: +1-604- 822-9185 E-mail: hussein@ece.ubc.ca 